\title{
EEG-Neurofeedback in psychodynamic treatment of substance dependence
}

\author{
Human F. Unterrainer ${ }^{1,2 *}$, Andrew J. Lewis ${ }^{3}$ and John H. Gruzelier ${ }^{4}$ \\ ${ }^{1}$ Centre for Integrative Addiction Research (Grüner Kreis Society), Vienna, Austria \\ ${ }^{2}$ Department of Psychology, Karl-Franzens-University, Graz, Austria \\ ${ }^{3}$ Faculty of Health, School of Psychology, Deakin University, Melbourne, VIC, Australia \\ ${ }^{4}$ Department of Psychology, Goldsmiths, University of London, London, UK \\ ${ }^{*}$ Correspondence: human.unterrainer@uni-graz.at \\ Edited by: \\ Nikolai Axmacher, University of Bonn, Germany \\ Reviewed by: \\ Juergen Fell, University of Bonn, Germany
}

Keywords: neuropsychoanalysis, neurofeedback, psychodynamic psychotherapy, addiction, anhedonia, theta, SMR

\section{A commentary on}

EEG-neurofeedback and psychodynamic psychotherapy in a case of adolescent anhedonia with substance misuse: Mood/theta relations

by Unterrainer, H. F., Chen, M. J., and Gruzelier, J. H. (2013). Int J Psychophysiol. pii: S0167-8760(13)00061-5. doi: 10.1016/j. ijpsycho.2013.03.011

The relationship between therapeutic techniques and psychoanalytic theory is complex and defies a direct translation from theory to practice. However, especially in recent years, there have been increased efforts to bolster psychodynamic research by drawing on neuroscientific findings. Proponents of this approach argue that the so-called "neuropsychoanalytic" models are only drawing upon Freud's own originally neurological framework- reflecting one of the central disciplinary origins of psychoanalysis (Kaplan-Solms and Solms, 2000). Indeed, Freud considered his psychologically based models a temporary structure which would later be reinforced once mechanistic neurological processes were identified which corresponded to the psychological processes he described. While not entering the specifics of this claim, it is reasonable to suggest that the brain-mind relationship has always been fundamental to psychoanalysis but this relationship has not been exploited within its clinical techniques.

One means of further exploring the real time relationship between psychodynamic clinical processes and their manifestation in neuroprocessing is the integration of neurofeedback into psychodynamic sessions. Practically speaking, both interventions could be integrated, or at least inform one another. How this could be done is yet to be established.

We would recommend that the interventions are done in parallel, for the neurofeedback protocols address cognitive dysfunction sensory-motor rhythm (SMR) and well-being (A/T) so that more is gained from the psychotherapy which is necessary from the outset to provide psychodynamic support. For logistical reasons all three were done in an afternoon in our study but with hindsight this proved too taxing and separate sessions for all three would be recommended. Neurofeedback (NF) can be characterized as a form of instrumental learning using feedback in real time from the brain's electrical activity. Participants learn to regulate their brain activity themselves through a process of repetition and reinforcement (Gruzelier, 2012). NF training has been shown to be an effective tool to aid in the treatment of a wide range of psychiatric disorders. For one example, a substantial body of research has been conducted over the past three decades by Peniston using a slow EEG-wave protocol for the treatment of addictive disorders (Peniston and Kulkovsky, 1999). This "PenistonProtocol" became very popular and widely accepted as a research paradigm and has shown to be effective in a number of studies (Scott et al., 2005; Sokhadze et al., 2008). Substance use disorders result in specific alteration in brain activity that is detectable with the use of quantitative electroencephalography (EEG) methods (Peled, 2008). Psychodynamic psychotherapy aims to identify and modify these enduring patterns of thoughts, feelings, impulses, and defenses that, in turn, lead to maladaptive compromises, ineffective behavior, and conflicts in interpersonal relations (Shafranske, 2009). So the therapeutic question is in which way the altered brain activity can be normalized by either NF, psychodynamic therapy or the combination?

To further illustrate this we refer here to very limited data coming from a single case treatment of a student who, on a weekly basis throughout a university term, was given short-term psychodynamic psychotherapy and two EEG-neurofeedback protocols: SMR and alpha/theta (A/T) (Unterrainer et al., 2013). Substance misuse produces a diversity of EEG irregularities; there is no characteristic pattern. Accordingly neurofeedback therapy has focused on remedying the cognitive and affective deficits such as attention, impulsivity, anhedonia, etc. Our case study approach was informed by Scott et al. (2005) who preceded $\mathrm{A} / \mathrm{T}$ training with the a course of SMR training (see Egner and Gruzelier, 2001) reporting a beneficial outcome from the combined neurofeedback protocols on impulsive errors and reaction-time variability in a sustained attention task, aside from a reduction in alienation, depression and defensiveness. Here we attempted to treat a long term drug misuse habit co-occurring with a depressive mood disorder (Lewis et al., 2008). In this single case psychodynamic psychotherapy 
sessions were always applied right before the Neurofeedback training on the same day. As a result the patient's capacity for attention as evinced by application to course work in English literature, improved substantially, and there was a striking reduction in psychopathology. The improvement was much more rapid than we would have anticipated from either therapy alone. This tentative finding suggests a number of avenues for further exploration. For example, given the increased interest in the neurobiological foundations of psychoanalytic theory, EEG-neurofeedback could potentially also be used to understand therapeutic change processes (Linden, 2006). Exactly how that would be achieved, would require a repeating combined NF/ psychodynamic treatment approach in order to observe, how the NF learning outcome differs between positive and negative therapy outcomes. In order to confirm the interaction effect of these combined therapeutic interventions a randomized controlled trial would be necessary. Another recommendation would be to schedule the psychotherapy and neurofeedback sessions on separate days and also schedule the SMR and A/T protocols on different days. There are studies from other groups which have tried to combine neurofeedback and psychotherapy (Arns et al., 2009), however, to our knowledge this case is the first one, in which Neurofeedback was related to a strictly psychodynamic approach and certainly the first for drug misuse. Hopefully future studies will confirm our initial impression in order to further develop a NF informed model of psychodynamic psychotherapy, and to apply this beyond cases of substance misuse.

\section{REFERENCES}

Arns, M., de Ridder, S., Strehl, U., Breteler, M. and Coenen, A. (2009). Efficacy of neurofeedback treatment in ADHD: the effects on inattention, impulsivity and hyperactivity: a metaanalysis. Clin. EEG Neurosci. 40, 180-189. doi: $10.1177 / 155005940904000311$

Egner, T., and Gruzelier, J. H. (2001). Learned self-regulation of EEG frequency components affects attention and event-related brain potentials in humans. Neuroreport 12, 4155-4159. doi: 10.1097/00001756-200112210-00058

Gruzelier, J. H. (2012). "Enhancing imaginative expression in the performing arts with EEG-neurofeedback," in Musical Imaginations: Multidisciplinary Perspectives on Creativity, Performance and Perception, eds D. Miell, R. MacDonald, and D. Hargreaves (Oxford: Oxford University Press), 332-350.

Kaplan-Solms, K., and Solms, M. (2000). Clinical Studies in Neuro-Psychoanalysis. Madison, CT: International Universities Press, Inc.

Lewis, A. J., Dennerstein, M., and Gibbs, P. M. (2008). Short-term psychodynamic psychotherapy: review of recent process and outcome studies. Aust. N.Z. J. Psychiatry 42, 445-455. doi: 10.1080/00048670802050520

Linden, D. E. J. (2006). How psychotherapy changes the brain-the contribution of functional neuroimaging. Mol. Psychiatry 11, 528-538. doi: 10.1038/sj.mp.4001816

Peled, A. (2008). Neuroanalysis: Bridging the Gap Between Neuroscience, Psychoanalysis and Psychiatry. East Sussex: Routledge.

Peniston, E. G., and Kulkovsky, P. J. (1999). "Neurofeedback in the treatment of addictive disorders," in Introduction to Quantitative EEG and Neurofeedback, eds A. Abarbarnel and J. R. Evans (London: Academic Press), 157-179. doi: 10.1016/B978-012243790-8/50008-0

Scott, W. C., Kaiser, D., Othmer, S., and Sideroff, S. I. (2005). Effects of an EEG biofeedback protocol on a mixed substance abusing population. Am. J. Drug Alcohol Abuse 31, 455-469. doi: 10.1081/ADA200056807

Shafranske, E. P. (2009). Spiritually oriented psychodynamic psychotherapy. J. Clin. Psychol. 65, 147-157. doi: 10.1002/jclp.20565

Sokhadze, T. M., Cannon, R. L., and Trudeau, D. L. (2008). EEG-Biofeedback as a treatment for substance use disorders: review, rating of efficacy and recommendations for further research. Appl. Psychophysiol. Biofeedback 33, 1-28. doi: 10.1007/s10484-007-9047-5

Unterrainer, H. F., Chen, J. L., and Gruzelier, J. H. (2013). EEG-neurofeedback and psychodynamic psychotherapy in a case of adolescent anhedonia with substance misuse: $\operatorname{mood} /$ theta relations. Int. J. Psychophysiol. doi: 10.1016/j.ijpsycho.2013. 03.011. [Epub ahead of print].

Received: 27 June 2013; accepted: 12 September 2013; published online: 01 October 2013.

Citation: Unterrainer HF, Lewis AJ and Gruzelier JH (2013) EEG-Neurofeedback in psychodynamic treatment of substance dependence. Front. Psychol. 4:692. doi: 10.3389/fpsyg.2013.00692

This article was submitted to Psychoanalysis and Neuropsychoanalysis, a section of the journal Frontiers in Psychology.

Copyright (c) 2013 Unterrainer, Lewis and Gruzelier. This is an open-access article distributed under the terms of the Creative Commons Attribution License (CC BY). The use, distribution or reproduction in other forums is permitted, provided the original author(s) or licensor are credited and that the original publication in this journal is cited, in accordance with accepted academic practice. No use, distribution or reproduction is permitted which does not comply with these terms. 